\title{
Análisis de la Productividad en la Construcción de Vivienda basada en Rendimientos de Mano de Obra
}

\section{Analysis of Productivity in Housing Construction Based on Labor Performance}

DOI: http://dx.doi.org/10.17981/ingecuc.12.1.2016.02

Artículo de investigación científica - Fecha de recepción: julio 29 de 2015 - Fecha de aceptación: 04 de noviembre de 2015

\author{
Adriana Gómez Cabrera \\ Magíster en Ingeniería Civil, Pontificia Universidad Javeriana. Bogotá (Colombia). \\ adrianagomez@javeriana.edu.co \\ Diana Carolina Morales Bocanegra \\ Magíster en Ingeniería Civil, Pontificia Universidad Javeriana. Bogotá (Colombia). \\ dmoralesb@javeriana.edu.co
}

Para citar este artículo / To reference this paper:

A. Gómez Cabrera y D. Morales Bocanegra, "Análisis de la productividad en la construcción de vivienda basada en rendimientos de mano de obra", INGE CUC, vol. 12, no. 1, pp. 21-31, 2016. DOI: http://dx.doi.org/10.17981/ ingecuc.12.1.2016.02

\begin{abstract}
Resumen-- El presente trabajo se basa en la identificación y eliminación de pérdidas dentro del proceso constructivo de edificaciones en la ciudad de Bogotá con el fin de aumentar la productividad. La metodología para recolección de información fue el muestreo de campo con el apoyo de imágenes digitales. Esto permitió identificar los principales factores causantes de pérdidas, entre los que se encuentran: esperas de material, desplazamientos, reprocesos, clima, entre otros. También se realizaron encuestas al personal de obra para identificar aspectos de percepción motivacional de los trabajadores y otros aspectos generales de la obra que afectaban la productividad, como condiciones de salario, clima laboral, estado de ánimo, entre otros. Finalmente, se llevó a cabo una simulación digital para plantear escenarios de mejoramiento donde se identificaban impactos de acciones de mejora que fueron diseñadas de acuerdo con los resultados encontrados en la etapa inicial.
\end{abstract}

Palabras claves-- Construcción sin pérdidas; simulación de procesos constructivos; productividad de mano de obra en construcción.

\begin{abstract}
This paper is focused on the identification and elimination of waste in building construction process in the city of Bogota so as to increase productivity. The methodology for gathering information was direct observation supported by digital images. Common causes of construction waste were identified, such as insufficient materials, inefficient material distribution, rework, and weather, among others. The data collection method also included construction workers interviews to identify motivational aspects and other general issues that affected productivity. Factors like salaries, workplace environment and mood were identified. Finally, discrete event simulation models were created for the real scenario and different scenarios were proposed.
\end{abstract}

Keywords-- Lean Construction; Construction Process Simulation; Construction Labor Productivity. 


\section{INTRODUCCIÓN}

Actualmente en la industria de la construcción es cada vez más común escuchar conceptos relacionados con la productividad, ya que debido a la globalización es una necesidad para las empresas ser cada vez más productivas y competitivas para así garantizar su permanecía en el tiempo. En Colombia, la construcción es uno de los principales motores de la economía, por lo cual este sector está en búsqueda de métodos que permitan planear y desarrollar proyectos eficientes, que no incurran en sobrecostos ni reprocesos y que disminuya su nivel de incertidumbre [1]. Dentro de estos métodos se encuentran la filosofía de Lean construction cuya función principal es buscar la optimización de los recursos, costos y tiempos, orientando sus resultados a la identificación y eliminación de pérdidas y todo aquello que no genere valor dentro del proceso de producción [2]. Muchas de estas pérdidas están principalmente dadas por el recurso humano, quien se ve afectado por ciertos factores que en general no están identificados y su impacto no se encuentra cuantificado [3].

En este proyecto de investigación se identificaron algunos de esos factores que están relacionados con la mano de obra por medio del método del muestreo de campo, toma de videos en tres proyectos de construcción objeto de la investigación y entrevistas a los trabajadores para conocer los factores de percepción motivacional que no son fácilmente identificables y que pueden estar relacionados con la productividad. Finalmente, por medio del software de simulación Arena de Rockwell software, se realizó una simulación del proceso constructivo de una de las obras en su etapa de estructura involucrando actividades de armado de acero, colocación de formaleta y vaciado de concreto. Esta simulación se realizó con los datos recopilados y con el fin de predecir el comportamiento del modelo al eliminar o disminuir los factores que fueron identificaron como pérdidas y que afectan la productividad. Teniendo en cuenta que la simulación es una herramienta para la gestión del conocimiento generando modelos de procesos constructivos es posible considerar en los mismos lecciones aprendidas en cada proyecto [4]. Por último, de acuerdo con lo observado y con los resultados de la simulación se plantearon unas recomendaciones según los aspectos y factores encontrados en la investigación.

Estas recomendaciones servirán de referencia para proyectos futuros de investigación y a las empresas constructoras que quieran aplicar los procesos lean es sus empresas. Existen factores que afectan la productividad en los procesos de construcción, por lo cual surge la necesidad de conocer y profundizar en cuáles son estos factores. Al identificar estos aspectos es posible analizarlos y actuar sobre ellos para así proponer acciones de mejora y optimizar cada vez más los procesos productivos. Esta información y conocimiento serán útiles en la estimación de costos y tiempos de un proyecto. Teniendo datos reales de rendimientos de mano de obra y de los problemas más comunes que afectan este rendimiento, se pueden lograr estudios de costos que indiquen la viabilidad real de un proyecto al momento de la planeación, esto con el fin de aumentar la productividad del personal, reducir los tiempos de ejecución del proyecto y a la vez los costos. Con las observaciones realizadas en campo se analizará la productividad con respecto a factores como tiempos, movimientos, utilización de recursos, distribución de cuadrillas, etc. del proceso, lo cual también permite realizar observaciones acerca de seguridad industrial y la prevención de riesgos, logrando así que se mejoren las condiciones de los trabajadores al interior de la obra [5]. Por lo anterior resulta importante conocer y cuantificar los factores que afectan directamente la mano de obra, entre otras para:

1. Aumentar el conocimiento de la mano de obra y de su situación como ser humano dentro de la obra, así como su desempeño en el trabajo.

2. Generar el conocimiento de la productividad actual de los proyectos.

3. Crear estrategias de mejora para aumentar la productividad a partir del recurso humano que estén dirigidas desde la gerencia de la obra.

4. Mejorar las condiciones de trabajo de todos los participantes dentro de la obra.

En la siguiente figura se enmarca la importancia de enfocar los procesos hacia el mejoramiento de la productividad:

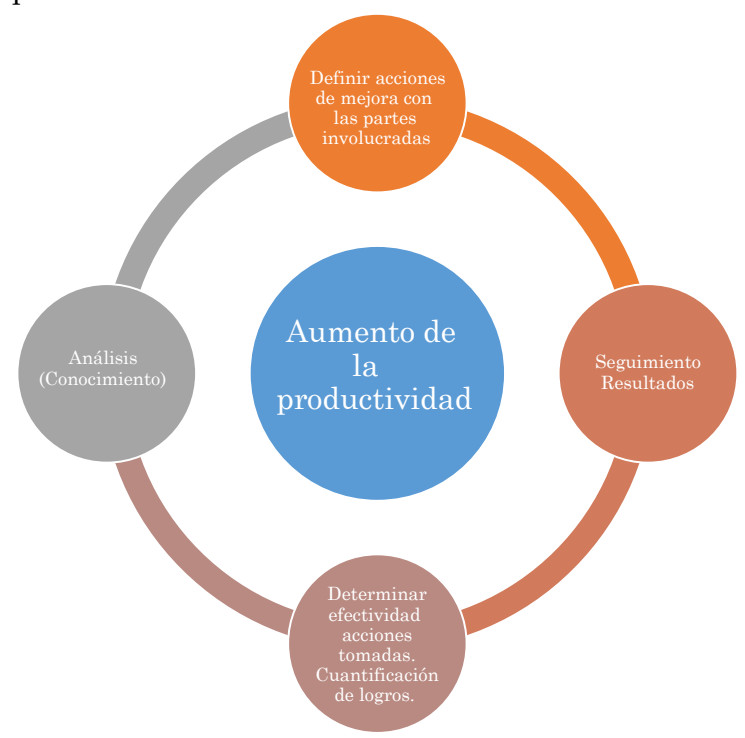

Fig. 1. Importancia del conocimiento de la productividad. Fuente: Autores.

Existen datos de la aplicación teórica de mejoramiento de la productividad pero la mayoría son producto de investigaciones académicas, ya que en la industria de la construcción no se tienen metodo- 
logías claras. Es por eso que por medio de la simulación se busca predecir estos escenarios óptimos, midiendo los tiempos de duración de las actividades y determinando los factores fundamentales causantes de las pérdidas de tiempo que generan bajas en la productividad [6]. Se tienen registros de bajos niveles de productividad en el sector de la construcción [7], soportados también por estudios realizados con técnicas como el muestreo de trabajo, entre otras. Estas técnicas permiten conocer la forma en que los obreros invierten su tiempo para el desarrollo de las actividades.

Es así como en general el sector de la construcción (aunque puede variar por actividad) presenta porcentajes de trabajo no productivo de cerca del $40 \%$, el tiempo restante se ve invertido en la realización de actividades de desarrollo y apoyo de la actividad principal. Este alto porcentaje que no contribuye a la realización de la actividad principal es considerado como una pérdida, gasta recursos y tiempo y no aportan valor. Particularmente en la construcción, la productividad presenta bajas investigaciones y bajos niveles de aumento respecto a otros sectores económicos: la manufactura ha presentado un aumento en la productividad del $100 \%$ en los últimos 30 años, mientras que la construcción se mantiene igual [8].

El sector de la construcción es para Colombia, uno de los sectores más importantes por la generación de empleo y por su crecimiento anual. En los últimos cinco años el porcentaje de PIB aportado por el sector es en promedio $7.4 \%$, pero durante el primer trimestre de 2013, el sector de la construcción representó 8,2\% del total del Producto Interno Bruto (PIB) de Colombia, mientras que otros sectores (aproximadamente 40 en total) tienen un promedio de $2 \%$ [9]. De otro lado, el recurso humano es el más importante para la ejecución de una obra y es quien, con su rendimiento, varía el rendimiento de los otros recursos [10], [11]. Sin embargo y de acuerdo con los análisis realizados por el Consejo Privado de Competitividad, una de las brechas de productividad está dada por el recurso humano, quien desafortunadamente se ve afectado por diferentes factores que reducen directamente los niveles de productividad. En la búsqueda de la medición de la productividad se han diseñado herramientas y estudios en los que se identifiquen estas falencias, ya que se plantea que al identificar dentro del proceso constructivo las causas de estas pérdidas y reduciéndolas, hay un incremento en la productividad [5].

Las áreas de mayor impacto en el mejoramiento de la productividad se centran en la planeación, la gerencia y construcción, y no necesariamente implica un mayor costo, consumo de tiempo u otras dificultades. Sin embargo, se requiere de compromiso para identificar las áreas a mejorar para aplicar las modificaciones de mejora y mantenerlas en el tiempo [12], [13].
Aunque muchos de los factores que afectan la productividad están relacionados directamente con el recurso humano, no en todos los casos se brinda la importancia que merece, teniendo en cuenta que la mano de obra es un componente fundamental del proceso productivo; es por eso que en la presente investigación se profundiza un poco más sobre el tema, y con la ayuda de la simulación digital se predice la afectación que estos factores tienen sobre la productividad. De esta forma, se plantea una herramienta útil para la toma de decisiones con respecto a los métodos constructivos y recursos involucrados con el fin de minimizar su costo y duración. Aunque las investigaciones sobre simulación en la industria de la construcción están avanzando académicamente, su aplicación en el sector aún es limitada debido a que se sigue trabajando con las técnicas tradicionales que a menudo son insuficientes. Algunas de las herramientas que se han utilizado para simulación en construcción son CYCLONE (1977), INSIGHT (1987), RESQUE (1987), UM-CYCLONE (1989), Micro-CYCLONE (1990), STROBOSCOPE (1994), ABC (1999) y HK CONSIM (2003) [14].

Dentro de las investigaciones referentes al tema, vale la pena resaltar la realizada por [15] donde se definen los conceptos y aplicaciones de la nueva filosofía de la construcción llamada Lean construction cuya teoría se basa en la búsqueda de la optimización de recursos, tiempos y costos. Esta metodología ha sido implementada en muchas empresas a nivel internacional desde 1993 y se basa en la eliminación sin desperdicios o pérdidas, como aquellas actividades que no generan valor sino que, por el contrario, causan re-procesos y demoras en la cadena productiva. El mejoramiento de esta productividad consiste en la identificación de las causas que originan esas pérdidas para proponer acciones de mejora y así, al tenerlas identificadas y al conocer estos factores que la afectan tanto positiva como negativamente, se puede actuar sobre ellas haciendo las respectivas correcciones y acciones de mejora que permitan, con el debido seguimiento, ir aumentando la productividad en los proyectos de construcción.

En el trabajo realizado por [12], que consistió en una investigación sobre rendimientos y mano de obra empleados en actividades para la construcción de vivienda de interés social en mampostería estructural, se tomaron datos reales medidos en obra para conformar una base de datos y se identificaron factores que afectaban esta mano de obra para posteriormente utilizar un software que permitiera predecir este consumo a partir de la correlación de los diferentes factores. Como conclusión de ese estudio se tienen las siguientes premisas: los sobrecostos a los que se ven asociados los proyectos por una mala identificación y cuantificación de los rendimientos es bastante elevado; el modelo planteado se ajusta a ciertas condiciones propias de un proyecto de VIS; la investigación da unos consumos 
estándares que se pueden aplicar a otros proyectos; las ecuaciones presentadas muestran una correlación significativa entre el consumo y algunas variables independientes, por lo cual son estadísticamente válidos. Sin embargo, se limita a dos obras en la ciudad de Medellín y pueden no ser suficientes para generalizar la metodología, tampoco se realizó un análisis de impacto de las variables tiempo y costo en el rendimiento de la mano de obra. Un futuro trabajo realizó la implementación conjunta de la simulación de operaciones, y la técnica de líneas de balance permitió la comparación y la toma de decisiones con menor incertidumbre que la proporcionada a través del conocimiento empírico [12].

Otros investigadores realizan una estimación de la productividad real de una obra para un caso específico por medio del método de "Muestreo del trabajo". En este trabajo se dan unos valores promedio de caracterización del trabajo productivo, contributivo y no contributivo, también plantea que al identificar y eliminar los problemas que afectan la productividad, se reducirán los costos asociados. El trabajo se realizó a partir un caso puntual en Argentina, pero las prácticas pueden ser extendidas a otras obras [16]. Diferentes estudios de caso generan una serie de recomendaciones para disminuir el tiempo total de la construcción dando a los encargados de la planeación de los proyectos las herramientas necesarias que permitan evaluar varios escenarios de manera probabilística, lo cual permitirá una mejor toma de decisiones y una disminución de la incertidumbre del comportamiento del proyecto [17]. El tema también ha sido abordado por [18], donde a partir del levantamiento de información en campo para un proceso constructivo de estructura se empleó la simulación de eventos discretos. En esta investigación se diseñó un modelo de simulación en el software Arena, el cual permite controlar la duración de las actividades, consumo de materiales y la correcta utilización del recurso, logrando así, y de acuerdo con las características propias de la obra, introducir la información y datos de entrada para el modelo. Con esta herramienta, se pudieron simular varios escenarios antes de la puesta en marcha del proyecto, lo cual proporcionó varios instrumentos y lecciones aprendidas para una mejora continua del proceso constructivo. Como resultado, en una de las alternativas se obtuvo que al disminuir el personal de la obra en oficiales y ayudantes, se puede mantener la duración del proyecto, disminuyendo así los costos asociados a este recurso. Otro ejemplo de Colombia, analiza las actividades de mampostería y muro en concreto en la construcción de una edificación cuyo sistema estructural consistía en pantallas de concreto reforzado construido con formaleta manoportable. Se simularon escenarios y se generaron recomendaciones como: revisar el proceso de mampostería, ubicar el material más cerca de su ejecución, cambiar los vehículos para el transporte de material, adquirir baldes y rodillos con líquidos desencofrantes, garantizar la terminación de las actividades del día anterior, entre otros. Finalmente, al realizar el balance del trabajo de acuerdo con las duraciones y a la reubicación del personal, se concluyó que se podría hacer una reducción de 1 hora al día de trabajo [1].

Siguiendo esta misma línea de investigación, en el trabajo realizado por [6] se presenta una propuesta mediante el uso de tecnología para capturar imágenes que permitan medir la productividad, y que por medio de la aplicación de los conceptos de la filosofía Lean construction se planteen unas posibles soluciones para mejorar estos aspectos que afectan el rendimiento. Esta investigación ofrece un sistema que facilita la toma de decisiones con respecto a métodos constructivos y los recursos involucrados para cada proyecto, todo esto con el objetivo de que dentro de los proyectos de construcción se logre aumentar los rendimientos y disminuir los costos asociados a éstos. Los resultados obtenidos dieron como resultado que al ser implementado se redujeron los tiempos de ciclo y se dio un mejor uso de los recursos, lo que se traduce en ahorro de recursos para el proyecto. De estos resultados obtenidos se plantearon nuevos escenarios teóricos que posteriormente se simularon y arrojaron como resultado un ahorro en tiempo y costos. Se concluye que una de las causas principales para las pérdidas de tiempo es que los materiales o los equipos no son entregados cerca de donde se van a utilizar y que la actividad en la que se utiliza más personal del necesario es en el vaciado de concreto de los elementos estructurales.

Otra investigación se centra exclusivamente en la productividad laboral del recurso humano con el fin de generar ahorros en tiempo y costo por medio de la administración adecuada de los mismos, además, relaciona la productividad con los conceptos de valor ganado. Asimismo, abarca las definiciones de construcción, productividad laboral, aspectos, medidas, factores y algunas técnicas que pueden ser utilizadas para la medición y técnicas de modelado. El resultado principal a partir de la literatura es que no hay una definición estándar de la productividad. Este estudio proporciona una guía para conocer los pasos necesarios para mejorar la productividad del trabajo de la construcción y, en consecuencia, del rendimiento del proyecto. Puede ayudar a mejorar el rendimiento global de los proyectos de construcción a través de la aplicación del concepto de puntos de referencia. Especialmente, enlista cinco técnicas comúnmente usadas para la medición de la productividad en obra, lo cual nos abre la posibilidad de estudiarlas y elegir la de mayor aplicación en el medio colombiano: técnica de muestreo, técnica de encuesta a empleados, técnica de estudio de tiempo, técnica de análisis de movimiento y técnica de tiempo en grupo [19]. 
Otro reciente trabajo relaciona la productividad como la estrategia para lograr una alta competitividad frente a otras empresas. Sin embargo, se hace énfasis en que para ello se requiere de un aumento de la productividad manteniendo constantes el precio y la calidad. Se indica que la mayoría de los estudios realizados muestran que en el lugar de trabajo los trabajadores tienen una productividad por debajo del $50 \%$, y se realiza un estudio con 25 directores de obra para definir cinco puntos sobre los cuales se puede aumentar la productividad con los cuales se desarrolló un modelo para hacer mejoras y soluciones alternativas que pueden servir para proyectos futuros [20].

La simulación computacional como herramienta fundamental para la planeación y ejecución de la obras civiles es propuesta por [21] en un caso de estudio que presenta los beneficios a largo plazo y plantea lineamientos para futuros desarrollos computacionales que sigan previendo y aportando a la planeación y ejecución de proyectos de construcción. Este estudio complementa muy bien la filosofía de simulación implementada en este trabajo ya que concluye que la simulación es el futuro de la planeación de los proyectos puesto que aportan al mejoramiento de los procesos constructivos.

Investigaciones más recientes presentan la simulación de la construcción de un hotel por medio de la simulación de eventos discretos. Este estudio se realizó por medio de la herramienta Tecnomatx plant simulation. El resultado obtenido arrojó que la simulación digital es complemento para las etapas de planeación y control durante la etapa de ejecución [22]. Una investigación colombiana también relaciona la simulación como una herramienta que no debe utilizarse de manera aislada sino que, por el contrario, sus beneficios se maximizan al analizarla en conjunto con metodologías de modelación digital de edificaciones para integración de proyectos y con herramientas de optimización de programación de obra [23].

\section{Metodología}

Para la recolección de información se tomaron datos de tres obras en la ciudad de Bogotá. Las obras seleccionadas corresponden a uso residencial y construcción con sistema aporticado con muros de concreto reforzado. Estas obras son de una misma constructora con características similares en su proceso de ejecución. El proyecto 1 consta de 2 torres de 16 y 26 pisos; el sistema constructivo consiste en un sistema convencional aporticado en muros y losas de concreto reforzado. El proyecto 2 consta de 4 torres de 22 pisos y el sistema constructivo utilizado para este proyecto es el sistema de pórticos que forman un conjunto de vigas y columnas que se conectan rígidamente; el concreto se producía en obra mediante una planta mezcladora. El proyecto
3 consta de 4 torres de apartamentos de 16 pisos y el sistema estructural es aporticado con estructuras de muros y placas en concreto reforzado. Para los 3 proyectos, la metodología empleada se resume en la Fig. 2

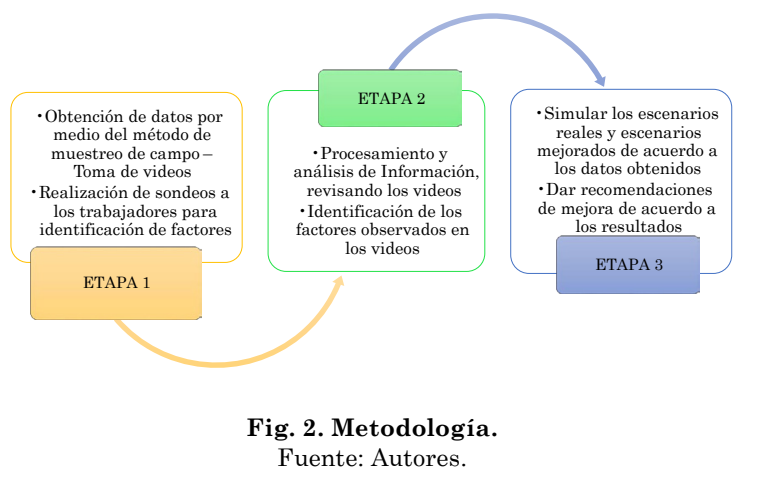

\section{A. Trabajo de campo y recolección de información}

La obtención y captura de la información se realizó por medio del método de muestreo de campo. Se realizaron visitas a cada una de estas obras en las que se tomaron videos de las actividades que ejecutaban los trabajadores durante diferentes horas del día. Lo anterior con el fin de tener acceso a las actividades en diferentes horas y días de la semana, y así medir e identificar las variaciones en diversas condiciones. Se tomaron datos durante las actividades de armado de acero, instalación de formaleta y vaciado de concreto para la elaboración de columnas, placa y vigas. La Fig. 3 muestra un diagrama con los procesos macro y las actividades en cada uno de ellos:
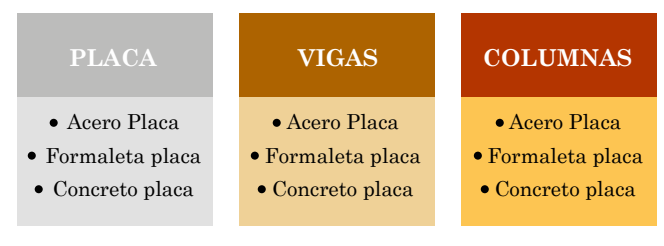

Fig. 3. Procesos y actividades de la medición. Fuente: Autores.

Teniendo en cuenta que no todos los factores son identificables a simple vista, se realizaron entrevistas personalizadas a los trabajadores. Estas entrevistas permitieron tener un acercamiento a ellos para conocer y medir factores intrínsecos y que pueden tener un valor significativo en la afectación de la productividad y de su rendimiento laboral. En esta etapa se determinaron las preguntas a realizar a los trabajadores, las cuales se diseñaron con el fin de identificar esos posibles factores que les afectan. Durante las inspecciones visuales realizadas a la obra, también se identificó el flujo de trabajo con la secuencia de actividades y el personal involucrado en cada una de ellas. 


\section{B. Procesamiento y análisis de la información}

En la segunda etapa se llevó a cabo todo el procesamiento y análisis de la información, caracterizando los factores y las variables involucradas y que afectan la productividad de la mano de obra. Se realizó el análisis estadístico de la información y se cuantificó el impacto que tienen estas variables en la productividad, verificando la distribución y participación de las actividades involucradas en cada uno de los procesos. Se analizó la información y se obtuvieron datos de trabajo productivo, contributivo y no contributivo con los factores identificados en la fase anterior, revisando detalladamente los videos tomados en obra y clasificando las actividades en los tipos de trabajo anteriormente mencionados.

\section{Simulación}

Por último, se realizó una fase de análisis de los resultados obtenidos determinado la relación que existen entre las variables relacionando lo observado en campo con los rendimientos reales obtenidos en obra. En esta fase se realizó una simulación con el software Arena incluyendo los factores y variables de acuerdo con las condiciones encontradas. Luego se plantearon escenarios mejorados y se dieron recomendaciones a partir del análisis de la información.

\section{Resultados}

\section{A. Análisis de actividades de obra}

Luego del proceso de obtención de material digital y análisis de los videos tomados y de las encuestas realizadas en las diferentes obras, se recopiló toda la información en una base de datos donde los diferentes tiempos por actividad y tipo de procesos se pudieron organizar y presentar de manera gráfica para así lograr ver porcentajes, tendencias y cantidades de los aspectos más relevantes del estudio. En la Fig. 4 se presentan estos resultados mencionados con su respectivo análisis y en la Fig. 5 se identifican los principales tipos de trabajos observados en las tres obras.

\section{Participación Por tipo de Actividad}

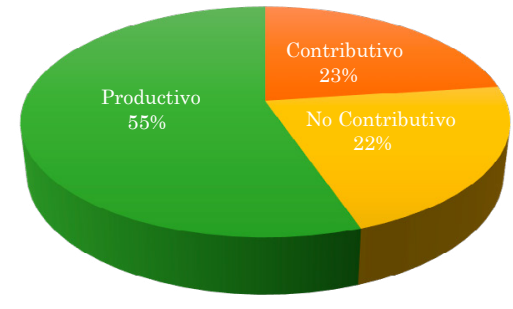

๓Contributivo $\llbracket$ No Contributivo $\square$ Productivo

Fig. 4. Participación por tipo de actividad. Fuente: Autores.

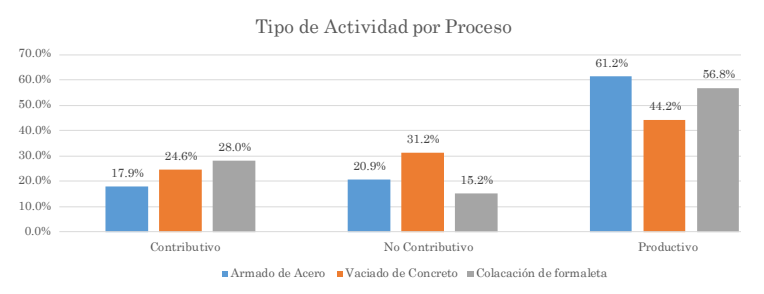

Fig. 5. Tipo de actividad por proceso. Fuente: Autores.

Como era de esperarse, el mayor porcentaje de tipo de actividad está comprendido en el trabajo productivo, pero es interesante ver que el $21.8 \%$, casi una cuarta parte de todo el tiempo, es gastado en actividades no contributivas, lo cual demuestra que las oportunidades de mejora para esta obra son muy significativas y que si se logra distribuir esas pérdidas de tiempo, ya sea en tiempo productivo o contributivo, se lograría disminuir los tiempos de los procesos o aumentar la calidad de éste, ya que se podría invertir más tiempo en control, medición y limpieza.

En la siguiente figura se puede observar que la actividad con mayor contribución de tiempo productivo es el armado de acero, demostrando que es la cuadrilla más eficiente.

A continuación se presenta la figura 6 con el detalle de aquellas actividades no contributivas, que representan la cuarta parte del tiempo total, como se mencionaba anteriormente.

Participación Actividades No Contributivas

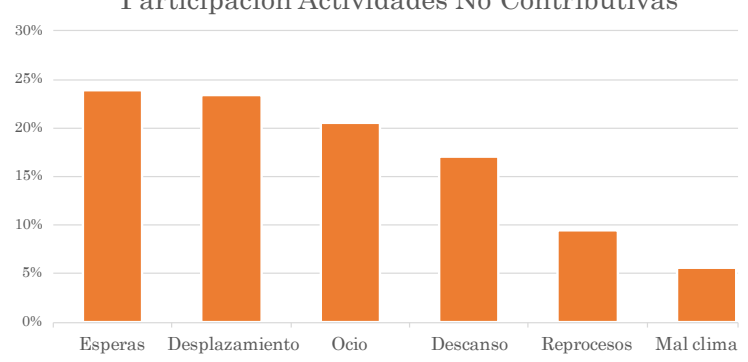

Fig. 6. Participación de las actividades no contributivas. Fuente: Autores.

Se puede observar que casi el $70 \%$ de las actividades no contributivas se dividen en tres aspectos: esperas, desplazamientos y ocio. Los dos primeros se pueden corregir desde una planeación administrativa más adecuada donde los puestos de trabajo y los recursos estén ubicados estratégicamente para evitar pérdidas de tiempo y de energía.

El ocio es un tema que se debe atacar directamente por los supervisores en piso y se debe identificar si está directamente ligada a factores motivacionales del personal o a una mala programación de actividades. Por otro lado, al detallar la distribución de actividades contributivas, se puede observar que la preparación de materiales tiene un valor importante con respecto a las otras, esto implica a las acti- 
vidades como: corte de acero, verificación de verticalidades, medición de formaletas, entre otras. Es importante resaltar que estas actividades son claves para garantizar una óptima calidad de la ejecución del trabajo.

Los datos también se pueden analizar desde otra perspectiva: detallando las actividades de tipo no contributivo pero para cada proceso constructivo estudiado en este trabajo; de esta manera, fácilmente se puede identificar el proceso que más genera pérdidas de tiempos y así poder, a su vez, proponer mejoras puntuales que afecten positivamente el proceso general de la obra. En la Tabla I se presenta la distribución porcentual de cada factor por actividad:

TABla I. CONTRIBuCión DE FACTORES POR ACTIVIDAD.

\begin{tabular}{|l|c|c|c|}
\hline \multirow{2}{*}{ Factor } & \multicolumn{3}{|c|}{ Actividad } \\
\cline { 2 - 4 } & $\begin{array}{c}\text { Armado } \\
\text { de acero }\end{array}$ & $\begin{array}{c}\text { Colocación de } \\
\text { formaleta }\end{array}$ & $\begin{array}{c}\text { Vaciado de } \\
\text { concreto }\end{array}$ \\
\hline Esperas & $20.6 \%$ & $17.8 \%$ & $31.1 \%$ \\
\hline $\begin{array}{l}\text { Desplaza- } \\
\text { miento }\end{array}$ & $25.5 \%$ & $27.5 \%$ & $18.7 \%$ \\
\hline Ocio & $7.8 \%$ & $27.3 \%$ & $23.9 \%$ \\
\hline Descanso & $21.0 \%$ & $16.9 \%$ & $12.9 \%$ \\
\hline $\begin{array}{l}\text { Re- } \\
\text { procesos }\end{array}$ & $17.0 \%$ & $10,5 \%$ & $7.0 \%$ \\
\hline Mal clima & $8.0 \%$ & $0.0 \%$ & $6.5 \%$ \\
\hline
\end{tabular}

Fuente: Autores.

Es interesante ver que para cada actividad se tiene un factor diferente liderando la pérdida de tiempos. En armado de acero resultan ser los desplazamientos los de mayor aporte con un $25.5 \%$ del total del tiempo no contributivo en esta actividad, mientras que para la colocación de formaleta el 37,7\% del total de pérdidas se deben al ocio. Finalmente, para el vaciado de concreto son las esperas las que más contribuyen a trabajos no contributivos con un $31.1 \%$.

Estos porcentajes no son muy dicientes si se comparan entre sí, ya que cada actividad tiene una participación diferente en la obra, por lo que se hace necesario utilizar la simulación para poder iden- tificar realmente qué factor es el que más influye en la pérdida de tiempos causando que los procesos sean improductivos. La tabla anterior se presenta también de manera gráfica en la Fig. 7 para poder visualizar fácilmente cómo es la distribución de los factores para cada actividad independiente.

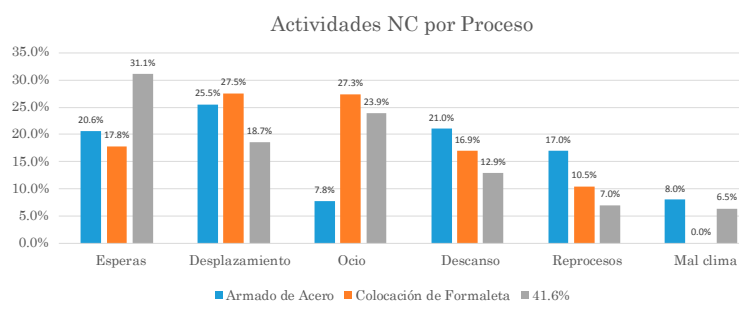

Fig. 7. Actividades no contributivas por proceso. Fuente: Autores.

Como resumen de los resultados generales de los proyectos se presenta la Fig. 8, donde se aprecia claramente cómo es la distribución de tipos de trabajos para cada obra.

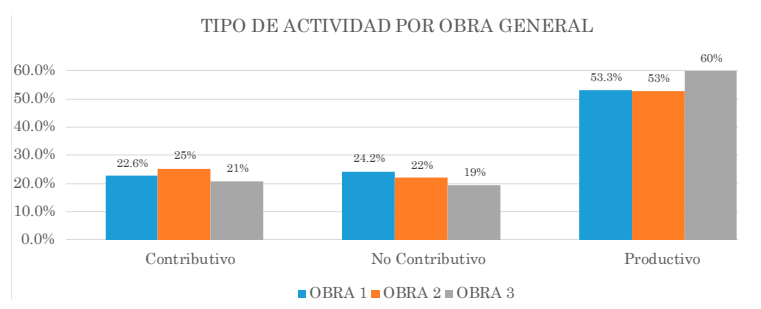

Fig. 8. Distribución de trabajo por obra. Fuente: Autores.

Para conocer la percepción que tienen directamente los trabajadores frente a su productividad, las inconformidades y factores que, de acuerdo con su percepción, afectan su desempeño dentro de la obra, se realizaron unos sondeos en los que se diseñaron una serie de preguntas para indagar y tratar de profundizar más sobre este tema. Como resumen general se presenta la Tabla II con las condiciones e inconformidades encontradas según los sondeos realizados. A nivel general se observa una gran inconformidad por parte de los trabajadores con respecto a los temas salariales, cambios constantes de actividad y trato por parte del jefe inmediato.

Tabla II. Factores de Percepción Motivacional.

\begin{tabular}{|c|c|c|c|c|c|}
\hline No & Percepción Motivacional & $\%$ & No & Percepción Motivacional & $\%$ \\
\hline 1 & Demora en el pago del sueldo; remuneración & $34 \%$ & 5 & Reconocimiento del trabajo & $8 \%$ \\
\hline 2 & Mal trato por parte del jefe y clima laboral & $15 \%$ & 6 & Problemas familiares & $7 \%$ \\
\hline 3 & Otros & $14 \%$ & 7 & La rutina & $6 \%$ \\
\hline 4 & Estado de ánimo y cansancio personal & $10 \%$ & 8 & Ninguna & $6 \%$ \\
\hline
\end{tabular}

Fuente: Autores. 


\section{B. Simulación}

Para conocer el comportamiento del proceso con las situaciones encontradas, se simuló en el software Arena el proceso constructivo de un ciclo de construcción de una torre de 7 pisos. Se analizó la construcción de cada piso como un ciclo del proceso, el cual tiene una duración aproximada de 19 días. Se determinaron tiempos de duración para las siguientes actividades para luego ingresar estos datos como datos de entrada para la simulación; para esto se contó con la colaboración de la empresa constructora quien suministró la información. Para la simulación se estableció el flujo de trabajo de las actividades, el cual se presenta a continuación en la Fig. 9.

Para la conceptualización del modelo se planteó una distribución por zonas, tal como se hace realmente en el proceso constructivo de la obra. En cada una de las cuatro zonas se llevan a cabo las actividades de armado de acero y encofrado en el mismo orden. Para esto se tuvo en cuenta la secuencia y las actividades que son predecesoras, tanto en la misma zona como en la combinación entre ellas. La combinación de zonas se refiere al proceso de vaciado de concreto que se simuló unificando la zona 1 y zona 2 , llamándola zona A, y luego la zona 3 y zona 4, llamándola zona B. Esto se presenta en la Fig. 10.
En estas dos nuevas zonas se realiza el proceso de vaciado de concreto y desencofrado. Este planteamiento del modelo hace que el diagrama de flujo en Arena sea un sistema en serie escalonado donde las actividades comienzan en la zona 1, y justo al acabar la primera actividad (encofrado de losa), arrancan simultáneamente esta primera actividad pero en la zona 2 y la segunda actividad (armado de malla electrosoldada) en la zona 1.

De esta manera se van escalonando las actividades hasta el punto que la zona 1 debe esperar a que la zona 2 esté lista para el vaciado de concreto, al igual que paso con las otras dos zonas. Una vez la zona A termina completamente las actividades, el modelo espera a que toda la zona $\mathrm{B}$ se finalice para dar por terminado el proceso del piso y arrancar por medio de un contador todo el proceso nuevamente pero con el siguiente piso. Este proceso se repite 7 veces (el número de pisos determinado) para dar por terminada la simulación y poder devolver la sumatoria de tiempos que equivalen al tiempo total de la construcción del edificio propuesto.

En la Fig. 11, se muestra cómo se planteó el modelo, aquí se pueden ver cómo van entrando las actividades y las zonas en la medida en que va avanzando la construcción.

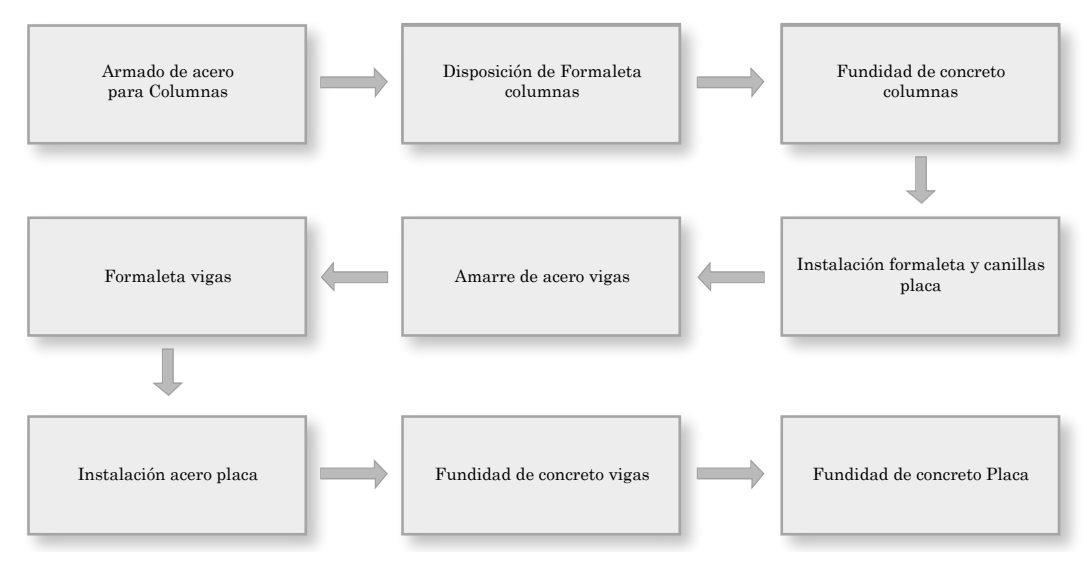

Fig. 9. Diagrama de flujo de trabajo.

Fuente: Autores.

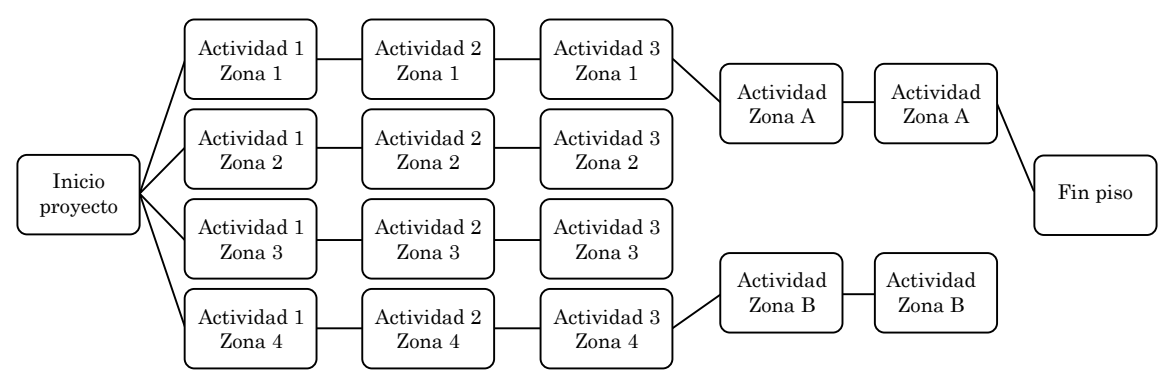

Fig. 10. Distribución de zonas en el proceso constructivo. Fuente: Autores. 


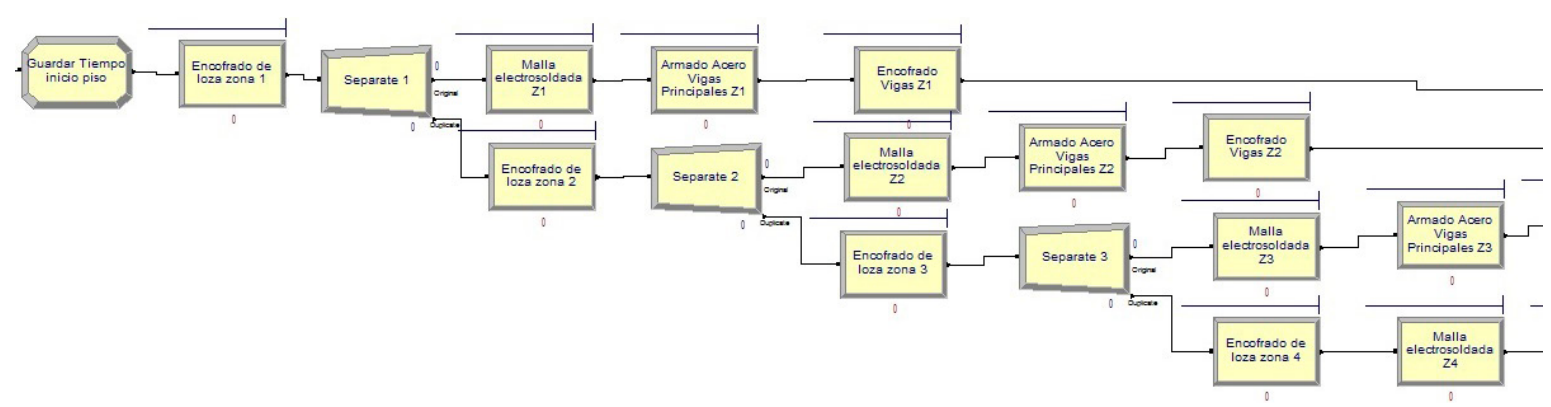

Fig. 11. Conceptualización del modelo.

Fuente: Autores.

\begin{tabular}{|c|c|c|c|c|}
\hline TNCEnconfrado (1) & \multicolumn{4}{|c|}{$(7+1.98 * \operatorname{BETA}(0.506,0.524)) *$ Porcentajes NC Form $(1) * \operatorname{Binarias}(1)$} \\
\hline TNCEnconfrado (2) & \multicolumn{4}{|c|}{$(7+1.98 * \operatorname{BETA}(0.506,0.524)) *$ Porcentajes NC Form $(2) * \operatorname{Binarias}(2)$} \\
\hline TNCEnconfrado (3) & \multicolumn{4}{|c|}{$(7+1.98 * \operatorname{BETA}(0.506,0.524)) *$ Porcentajes NC Form $(3) * \operatorname{Binarias}(3)$} \\
\hline TNCEnconfrado (4) & \multicolumn{4}{|c|}{$(7+1.98 * \operatorname{BETA}(0.506,0.524)) *$ Porcentajes NC Form $(4) *$ Binarias $(4)$} \\
\hline TNCEnconfrado (5) & \multicolumn{4}{|c|}{$(7+1.98 * \operatorname{BETA}(0.506,0.524)) *$ Porcentajes NC Form $(5) * \operatorname{Binarias}(5)$} \\
\hline TNCEnconfrado (6) & \multicolumn{4}{|c|}{$(7+1.98 * \operatorname{BETA}(0.506,0.524)) *$ Porcentajes NC Form $(6) * \operatorname{Binarias}(6)$} \\
\hline \multirow{2}{*}{\multicolumn{5}{|c|}{$\begin{array}{l}\text { TNCEnconfrado(1) } \\
\text { TNCEnconfrado(1)+TNCEnconfrado(2) }\end{array}$}} \\
\hline & \multicolumn{4}{|c|}{ TNCEnconfrado(1)+TNCEnconfrado(2) } \\
\hline \multicolumn{5}{|c|}{ TNCEnconfrado(1)+TNCEnconfrado(2)+TNCEnconfrado(3) } \\
\hline \multicolumn{4}{|c|}{ TNCEnconfrado(1)+TNCEnconfrado(2)+TNCEnconfrado(3)+TNCEnconfrado(4) } & \\
\hline \multicolumn{4}{|c|}{ TNCEnconfrado(1)+TNCEnconfrado(2)+TNCEnconfrado(3)+TNCEnconfrado(4)+TNCEnconfrado(5) } & \\
\hline
\end{tabular}

Fig. 12. Ecuación del modelo para variación de los factores.

Fuente: Autores.

Luego de definir el orden de las actividades y la secuencia lógica de cada piso, se hizo la formulación de cada proceso teniendo en cuenta las distribuciones de tiempos calculados por cada actividad. En esta formulación se incluye la participación porcentual de cada proceso y se establecen los tiempos de los trabajos contributivos, productivos y no contributivos. La sumatoria de estos tiempos equivale al tiempo total por cada actividad que a su vez se va sumando con la secuencia de actividades para calcular el tiempo total por piso, y finalmente, al correr todo el modelo, el tiempo total de la obra. A continuación en la Fig. 12 se presenta un ejemplo de la formulación hecha en Arena para el cálculo de los tiempos totales por actividad y por zona.

\section{Planteamiento de escenarios}

A continuación se presentan los resultados del modelo, en los cuales se varió cada uno de los factores utilizando el vector binario mencionado anteriormente. Se plantearon siete escenarios, y en cada uno se eliminó uno de los factores para así ver individualmente el impacto de cada uno de ellos; también se planteó un escenario en el que se varía la afectación de los factores, no se eliminan pero sí se reduce su impacto.
Es importante aclarar que el clima, como factor no contributivo, se mantuvo siempre presente en todos los escenarios ya que es un factor que no se puede controlar a diferencia de los otros cinco factores hallados.

En el escenario 1 se eliminaron las esperas, dando como resultado una disminución del 10,8\% con respecto al tiempo real de ejecución. Luego, en el escenario 2 al variar los desplazamientos se obtuvo un $12.5 \%$ menos de tiempo total; un resultado muy similar al del escenario 1. Siguiendo con la eliminación total de un factor específico, se planteó un escenario 3 donde se elimina por completo el ocio. En este escenario se obtuvo un $16 \%$ total en reducción de tiempo, lo cual es muy interesante ya que el ocio es un factor que puede ser controlado en la obra y que gracias a la simulación es evidente que aporta un gran porcentaje en las pérdidas de tiempos. En el escenario 4 se eliminó el factor de descansos y la disminución de tiempo bajó a sólo un 8.4\%, que es casi la mitad de lo visto en el escenario anterior. En el escenario donde se eliminaron los reprocesos, escenario 5, sólo hubo una reducción del 1.3\%, lo cual deja de ser interesante al compararse con los otros escenarios. A continuación, se presenta la Tabla III, donde de forma matricial se muestra cada escenario con sus respectivos escenarios: 
TABLA III. Escenarios CON CADA FACTOR.

\begin{tabular}{|c|c|c|c|c|c|c|c|c|}
\hline Factores & Esc1 & Esc2 & Esc3 & Esc4 & Esc5 & Esc6 & Actual & Combinado \\
\hline Esperas & 0 & 1 & 1 & 1 & 1 & 0 & 1 & 0.5 \\
\hline Desplazamiento & 1 & 0 & 1 & 1 & 1 & 0 & 1 & 0.2 \\
\hline Ocio & 1 & 1 & 0 & 1 & 1 & 0 & 1 & 1 \\
\hline Descanso & 1 & 1 & 1 & 0 & 1 & 0 & 1 & 0.8 \\
\hline Reprocesos & 1 & 1 & 1 & 1 & 0 & 0 & 1 & 0.7 \\
\hline Mal clima & 1 & 1 & 1 & 1 & 1 & 1 & 1 & 1 \\
\hline Tiempo total horas & 992.4 & 973.1 & 934.9 & 1019.6 & 1097.6 & 581.2 & 1112.5 & 918.2 \\
\hline Tiempo por piso (h) & 141.7 & 139.0 & 133.5 & 145.6 & 156.8 & 83.0 & 158.9 & 131.2 \\
\hline Disminución \% tiempo total & $10.8 \%$ & $12.5 \%$ & $16.0 \%$ & $8.4 \%$ & $1.3 \%$ & $47.8 \%$ & & $17.5 \%$ \\
\hline Días obra & 124.1 & 121.6 & 116.9 & 127.5 & 137.2 & 72.7 & 139.1 & 114.8 \\
\hline Días por piso & 17.7 & 17.4 & 16.7 & 18.2 & 19.6 & 10.4 & 19.9 & 16.4 \\
\hline
\end{tabular}

Fuente: Autores.

El escenario 6 se propone como un proceso ideal que a su vez llega a ser irreal, ya que se establece que todos los factores menos el mal clima sean eliminados por completo. Este escenario limita todo el rango que existe de mejora, donde al plantear una reducción total de los factores no contributivos se lograría una disminución del $47.8 \%$ del tiempo total de la obra. Lograr una obra en la mitad de tiempo implicaría un ahorro económico gigantesco para una constructora. Finalmente, se plantea un último escenario donde se propone una disminución porcentual de cada factor. Esto representa un trabajo administrativo de mejoras donde, gracias a una buena planeación de los recursos de la obra, el manejo de materiales y la motivación del personal, se lograría disminuir parcialmente cada factor logrando mejoras significativas en los tiempos totales de la obra. En el escenario combinado se modifican los factores con reducciones entre el $20 \%$ y $50 \%$, dejando el ocio con los valores actuales ya que en el escenario 3 se vio la importancia que este factor tiene. Con la disminución combinada se logró proponer un $17.5 \%$ de disminución del tiempo total de la obra, pasando de 19.9 días por piso a sólo 16.4 días, lo cual es una ganancia muy significativa. La estructura de este simulador permite variar los factores según las proyecciones de cada obra para prever si las acciones a implantar se justifican en cuanto a reducción de tiempos o no.

\section{Discusión}

En diferentes estudios realizados sobre el tema de productividad y la utilización de la simulación como herramienta de análisis de información se ven resultados efectivos, de forma que se pueda predecir el comportamiento del sistema bajo ciertas condiciones y características especiales. Esto se ve reflejado en estudios como el realizado por [6] o como el realizado por [18], en el cual se plantea una metodología para disminuir personal, reduciendo así los costos asociados a estos recursos y optimizando el tiempo para disminuir la duración total del proyecto. Con respecto a los resultados obtenidos en la medición de tiempos por medio del método de muestreo de campo, se observan valores de tiempo productivo de $55.3 \%$, contributivo de $22.9 \%$ y no contributivo de $21.8 \%$, esto a su vez difieren de los arrojados en el trabajo de [6], donde el $31.2 \%$ es productivo, el $46.2 \%$ es contributivo y el $22.8 \%$ es no contributivo.

En el presente trabajo se hace énfasis en las actividades no productivas, ya que es el objeto de la investigación, y por medio de la simulación se identifica el comportamiento de éstas dentro del proceso productivo. En otras investigaciones se enfocan en los tiempos de duración de las actividades y en la distribución de los recursos y personal, se identifican las posibles causas de las pérdidas y se dan recomendaciones para disminuirlas (por ejemplo factores como la planeación, reorganizar el tema del desplazamiento por materiales, tener las herramientas adecuadas, mejorar el tema de seguridad industrial, entre otros), pero no se hace un análisis detallado del impacto y el comportamiento de los factores que afectan la productividad. 


\section{Conclusiones y Recomendaciones}

- Después de realizar el análisis de la información se obtuvo que los factores identificados fueron: esperas de materiales y equipos, ocio, desplazamiento por traslado de material, reprocesos, descansos y mal clima.

- Los principales factores de acuerdo con la percepción de los trabajadores fueron:

1. Estado de ánimo del trabajador y cansancio personal.

2. Falta de recursos suficientes para desempeñar su labor.

3. Demoras en el pago del salario.

4. Reconocimiento de su trabajo.

5. Mal trato por parte del jefe y clima laboral.

6. La rutina.

7. Cuando no llegan los materiales y se alarga la jornada.

8. Cambio de labor de su especialidad.

- Luego de ver los resultados y de acuerdo con lo observado, se puede concluir que hay una clara falta de planeación en las obras y esto se ve reflejado durante el proceso de ejecución. Por ejemplo, los baños y el almacén implican pérdidas de tiempo significativas que se traducen en sobrecostos y retrasos en la programación. Realizar un análisis de layout puede minimizar este tipo de impactos, ya que se planea la logística para tener la menor cantidad de pérdidas por desplazamientos.

- A nivel general, los trabajadores presentan inconformidades con respecto a sus condiciones laborales dentro las obras donde trabajan; se observa que estos factores están directamente relacionados con la productividad en la ejecución de las actividades. Se podría profundizar en futuras investigaciones sobre el tema realizando las correlaciones que tienen estos factores motivacionales y su afectación específica en la productividad para así tener un modelo que incluya estos factores.

- La simulación digital permite ver alternativas y evaluar un proceso antes de su puesta en marcha, teniendo así datos de duración de los proyectos más acertados con la realidad, evitando sobrecostos y pérdidas en la etapa de ejecución y disminuyendo su nivel de incertidumbre.

\section{REFERENCIAS BIBLIOGRÁFICAS}

[1] J. D. Céspedes Henao, "Mejoramiento de la productividad en construcción : time-lapse y simulación digital como herramientas de análisis," Universidad de los Andes, Bogotá, 2010.

[2] L. Koskela -, Application of the New Production Philosophy to Construction. Labopratory for urban planning and building design, 1992.
[3] A. Serpell and L. F. Alarcón, Planificación y control de proyectos. Ediciones Universidad Católica de Chile, 2009.

[4] A. Gómez, "Simulación de procesos constructivos," Revista ingeniería de construcción, 2010.

[5] L. F. Alarcon, S. Fuster, M. Mora, and D. Sossdorf, "Utilización de imágenes y videos digitales para el mejoramiento de la productividad y prevención de riesgos en operaciones de construcción," 2009.

[6] A. Gómez, J. Echeverry, X. Giraldo, C. Otalora, and M. L. Cano, "Mejoramiento de procesos constructivos a partir de un modulo programable para captura de imágenes y simulacion digital" Rev. Ing. Construcción, vol. 27, 2012.

[7] CAMACOL, "Agenda Compet Marzo 2008," 2008.

[8] M. Radosavljević and R. M. W. Horner, "The evidence of complex variability in construction labour productivity," Constr. Manag. Econ., vol. 20, no. 1, pp. 3-12, Jan. 2002.

[9] DANE, "Indicadores económicos alrededor de la construcción." 2014.

[10] A. Enshassi, S. Mohamed, and S. Abushaban, "Factors affecting the performance of construction projects in the Gaza strip," J. Civ. Eng. Manag., vol. 15, no. 3, pp. 269-280, Jan. 2009.

[11] J. Contreras, A. Gómez, and J. Castaño, "Balance de cuadrillas y flujo de trabajo como herramienta para el mejoramiento de procesos constructivos," Revista Escuela Colombiana de Ingeniería, no. 91, 2013.

[12] L. fernando Botero and H. Acevedo, "Simulación de operaciones y línea de balance," Ingeniería y ciencia, pp. 29-45, 2011.

[13] A. Gómez, M. Granados, and I. Pérez, "Improving construction material and equipment logistics via simulation," Ing. Y Compet., vol. 17, no. 1, pp. 95-94, 2015.

[14] S. Abourizk, "Role of Simulation in Construction Engineering and Management:," J. Constr. Eng. Manag., vol. $136,2010$.

[15] L. F. Botero, Construcción sin perdidas, 2nd ed. 2006.

[16] A. Cantú, J. Moreno, M. Gallina, and G. García, "Productividad real en obras civiles. Análisis de un caso," 2009.

[17] D. Echeverry, H. Paez, and H. Mesa, "Simulación digital de procesos de construcción de estructura en concreto: casos de estudio práctico en Bogotá," Revista ingeniería de construcción, 2008.

[18] A. Gomez, "Revista ingeniería de construcción - Simulación de procesos constructivos," Rev. Ing. Construcción, vol. 25,2010

[19] M. E. Shehata and K. M. El-Gohary, "Towards improving construction labor productivity and projects' performance," Alexandria Eng. J., vol. 50, no. 4, pp. 321330, Dec. 2011.

[20] A. Hammad, F. Vahdatikhaki, C. Zhang, M. Mawlana, and A. Doriani, "Towards the smart construction site: Improving productivity and safety of construction projects using multi-agent systems, real-time simulation and automated machine control," 2012, pp. 1-12.

[21] S. AbouRizk, D. Halpin, Y. Mohamed, and U. Hermann, "Research in Modeling and Simulation for Improving Construction Engineering Operations," J. Constr. Eng. Manag., vol. 137, no. 10, pp. 843-852, Oct. 2011.

[22] F. Berner, V. Kochkine, I. Habenicht, S. Spieckermann, and C. Vath, "Simulation in manufacturing planning of buildings," 2013, pp. 3306-3317.

[23] A. Gómez, J. Avila, and N. Quintana, "Simulación de eventos discretos y líneas de balance, aplicadas al mejoramiento del proceso constructivo de la cimentación de un edificio," Rev. Ing. y Cienc., vol. 11, no. 21, 2015. 